\title{
Vitaminas B12, B6, B9 e homocisteína e sua relação com a massa óssea em idosos
}

\section{VtaminsBD, BG, B9, andhomocyteineandtheir redzion with bonemassinthe ddaly}

\section{Resumo}

O número de idosos nos últimos anos tem apresentado incremento em todo o mundo, o que acaba gerando inúmeras preocupações com a saúde dessa população, já que nessa fase da vida as alterações fisiológicas os tornam mais propensos a doenças, principalmente as crônicas não-transmissíveis. A osteoporose, uma doença osteometabólica frequente nos idosos, torna-se alvo de importantes estudos, uma vez que suas consequências afetam tanto a saúde física quanto a psicossocial. Contudo, são diversos os fatores que predispõem à osteoporose, entre os quais a deficiência de vitamina B12, vitamina B9 (folato) e vitamina B6 (piridoxina). A carência dessas vitaminas eleva os níveis de homocisteína, que age interferindo nas ligações cruzadas do colágeno, resultando em diminuição da resistência óssea e, consequentemente, na osteoporose e fraturas ósseas tanto em homens quanto em mulheres. Nesse contexto, este artigo tem como objetivo realizar uma revisão de literatura nas bases de dados LILACS e PubMed, sobre a relação dos níveis séricos de vitamina B12, folato, vitamina B6 e homocisteína com a diminuição da massa óssea em idosos.

\section{Abstract}

The number of elderly in recent years has increased worldwide, which ends up causing numerous health concerns of this population, since in this stage of life the physiological changes make them more prone to disease, especially chronic non-communicable diseases. Osteoporosis, an osteometabolic disease common in the elderly, becomes the subject of important studies, since its consequences affect both the physical and psychosocial health. However, there are various factors that predispose to osteoporosis, including the deficiency of vitamin B12, vitamin B9 (folate) and vitamin B6 (pyridoxine). The lack of these vitamins increases homocysteine levels, which acts

Palavras-chave:

Homocisteína. Vitamina B12. Ácido Fólico. Vitamina B6. Densidade Óssea. Osteoporose relacionada a idade. Idoso.

Key words: Homocysteine. Vitamin B12. Folic acid. Vitamin B6. Bone Density. Osteoporosis related to age. Elderly.

\footnotetext{
Programa de Pós-graduação em Gerontologia Biomédica. Pontifícia Universidade Católica do Rio Grande do Sul. Porto Alegre, RS, Brasil.

2 Instituto de Geriatria e Gerontologia. Pontifícia Universidade Católica do Rio Grande do Sul. Porto Alegre, RS, Brasil.

3 Faculdade de Enfermagem, Nutrição e Fisioterapia. Pontifícia Universidade Católica do Rio Grande do Sul. Porto Alegre, RS, Brasil.
} 
interfering with collagen cross-links, resulting in decreased bone strength and hence in osteoporosis and bone fractures in both men and women. In this context, this paper aims to conduct a literature review in the databases PubMed and LILACS, on the relationship of serum vitamin B12, folate, vitamin B6 and homocysteine with decreased bone mass in the elderly.

\section{INTRODUÇÃO}

O número de idosos, em todo o mundo, tem apresentado crescimento, sobretudo nos países em desenvolvimento. No Brasil, este fenômeno também está sendo observado, especialmente desde o início da década de $60 .^{1,2}$ Essa modificação demográfica ocasiona inúmeras preocupações, pois é nessa fase da vida que ocorrem diversas alterações morfológicas, fisiológicas, bioquímicas e psicológicas, tornando o indivíduo mais propenso a doenças, o que aumenta sua susceptibilidade de morte. ${ }^{1-3}$

Dentre as doenças crônicas mais presentes no envelhecimento, a osteoporose tem sido apontada como uma das prioridades de saúde pública mundial, devido a sua alta prevalência e efeitos sobre a saúde física e psicossocial do idoso. ${ }^{4}$

A osteoporose é a doença osteometabólica mais frequente no idoso, acometendo em média 75 milhões de pessoas na Europa, Estados Unidos e no Japão, sendo mais comum nas mulheres. ${ }^{5,6} \mathrm{~A}$ partir do climatério, com a redução dos níveis de estrógeno, ocorre a precipitação da diminuição de massa óssea. ${ }^{7,8}$ Por outro lado, nos homens a osteoporose é a segunda doença mais comum, ficando atrás somente da doença pulmonar obstrutiva crônica. ${ }^{5,6}$

Importantes modificações nos hábitos diários, como uma alimentação equilibrada, tanto em macronutrientes como em micronutrientes, uma dieta rica em cálcio, além de atividade física regular e exposição solar adequada, atuariam como fatores preventivos da integridade óssea. ${ }^{9}$ No entanto, as atuais investigações sugerem que uma dieta rica em vitamina $\mathrm{B} 12$, vitamina $\mathrm{B} 6$ e folato ou suplementação dessas vitaminas reduziria de forma significativa os níveis plasmáticos de homocisteína, que atualmente tem sido apontada como um fator de risco para osteoporose e fraturas ósseas. ${ }^{10-12}$
Diante disso, este artigo teve como objetivo realizar uma revisão da literatura, nas bases de dados eletrônicas do LILACS e PubMed, sobre a associação dos níveis séricos de vitamina B12, vitamina B6, folato e homocisteína, com a diminuição da massa óssea em idosos.

\section{METODOLOGIA}

Foi realizado levantamento bibliográfico nas bases de dados eletrônicas LILACS (Literatura Latino-Americana e do Caribe em Ciências da Saúde) e PubMed (National Library of Medicine). Os descritores utilizados em português foram: homocisteína, vitamina B12, vitamina B6, folato, polimorfismo MTHFR, idoso, densidade mineral óssea e osteoporose. Em inglês, os descritores foram: bomocysteine, B12 vitamin, B6 vitamin, folate, MTHFR polymorphism, elderly, bone mineral density e osteoporosis.

Na busca na base de dados LILACS, não se encontrou nenhum artigo que verificasse a associação entre os níveis séricos de vitamina B12, vitamina B6, folato e homocisteína com a massa óssea e osteoporose em idosos. Assim, foram utilizados somente artigos que abordavam a osteoporose, o envelhecimento populacional e o metabolismo da homocisteína. Por meio da PubMed, foram encontrados 50 artigos que verificavam tal associação.

Os critérios de seleção foram artigos originais e de revisão bibliográfica, publicados no período de 1997 a 2009. Para verificar "associação entre a deficiência de vitamina B12, B6, folato e da hiper-homocisteinemia com a diminuição da massa óssea", foram utilizados somente artigos originais entre os anos de 2000 e 2009, exceto uma bibliografia pertencente ao ano de 1966, considerada referência para o tema. 


\section{REVISÃO DE LITERATURA}

Osteoporose

A osteoporose é uma doença sistêmica caracterizada pela diminuição da densidade mineral óssea (DMO), com deterioração da microarquitetura do tecido ósseo, resultando na perda de resistência e aumento do risco de fratura por fragilidade, principalmente na coluna vertebral, quadrile punho. ${ }^{4,6}$ Estimativas apontam que o número de fraturas osteoporóticas terá um aumento expressivo nos próximos 50 anos. Nos Estados Unidos, ocorrem cerca de dois milhões de fraturas ao ano e estima-se que, em 2025, esses números aumentem para três milhões de fraturas anuais. ${ }^{6,13}$

São diversos os fatores que predispõem ao risco de osteoporose e fraturas, entre eles: histórico familiar, sexo feminino, níveis reduzidos de estrógeno, tabagismo, alcoolismo, síndromes de má absorção, doenças crônicas, hirperparatireoidismo, fármacos (corticoides, heparina e anticonvulsivantes), deficiência de vitamina $\mathrm{D}$, dieta pobre em cálcio e sedentarismo. ${ }^{9,14}$

Atualmente, alguns estudos têm investigado a relação entre a vitamina B12, vitamina B9, vitamina B6 na integridade da massa óssea, já que a deficiência dessas vitaminas elevaria os níveis de homocisteína (hiper-homocisteinemia), que interfere nas ligações cruzadas do colágeno, resultando na diminuição da resistência óssea e, consequentemente, em osteoporose e fraturas ósseas. ${ }^{11,15-17}$

\section{Metabolismo da homocisteína}

A homocisteína é um aminoácido sulfidrílico formado a partir da desmetilação da metionina. A homocisteína é metabolizada por meio de duas vias: a de remetilação (dependente de vitamina B12 e ácido fólico) e a de transfuração (dependente de vitamina B6). ${ }^{18}$

Na remetilação, a homocisteína é convertida a mationina, pela enzima metionina sintetase, que requer vitamina B12 como co-fator. Nesse momento, ocorre a redução do 5,10 metilenotetra-hidrofolato, que tem como finalidade doar agrupamento metil à metionina sintetase. Essa reação é catalisada pela 5,10 metilenotetra-hidrofolato-redutase (MTHF). ${ }^{19}$ Contudo, a remetilação também ocorre por meio do metabolismo da betaína, que doa agrupamento metil para a metionina sintetase, com o objetivo de converter a homocisteína em metionina (figura 1).

Na transfuração, a homocisteína é convertida em cistationina, reação essa catalisada pela enzima cistationina B sintetase (CBS), que necessita de vitamina B6 como co-fator e, após, gerando cisteína e alfa-cetobutirato. ${ }^{20,21}$ Uma redução da atividade da CBS por deficiência de co-fator, que é a vitamina $\mathrm{B} 6$, ou por alguma mutação, pode levar a um aumento da homocisteína ${ }^{22}$ (figura 1). A cisteína, formada a partir da homocisteína, pode ser incorporada em enzimas e proteínas podendo ter seu grupo -SH convertido em pontes dissulfeto, necessárias para a força e estabilidade de muitas proteínas, inclusive do colágeno. ${ }^{22}$

Baines et al. ${ }^{22}$ avaliaram mulheres na pósmenopausa e constataram que aquelas que apresentavam baixa massa óssea tinham níveis séricos diminuídos de cisteína e um risco mais elevado de fratura óssea.

A deficiência de vitamina B12, vitamina B6 e folato dificulta a execução de diversas reações enzimáticas. A redução dos níveis dessas vitaminas impede o funcionamento da metionina sintase, CBS e MTHFR, aumentando as concentrações plasmáticas de homocisteína (figura 1). Evidências atuais mostram que $o$ excesso de homocisteína está relacionado com alterações na estrutura de proteínas, peroxidação lipídica, inflamação e danos ao DNA. ${ }^{20}$

A hiper-homocisteinemia é um fator de risco para a osteoporose e fraturas ósseas, pois atua bloqueando as reações da enzima lisil-oxidase, responsável pela síntese das ligações cruzadas do colágeno, que tem como função estabilizar as fibras de colágeno no osso, produzindo uma estrutura rígida e forte ao tecido ósseo. ${ }^{23,24}$ (figura 1). 


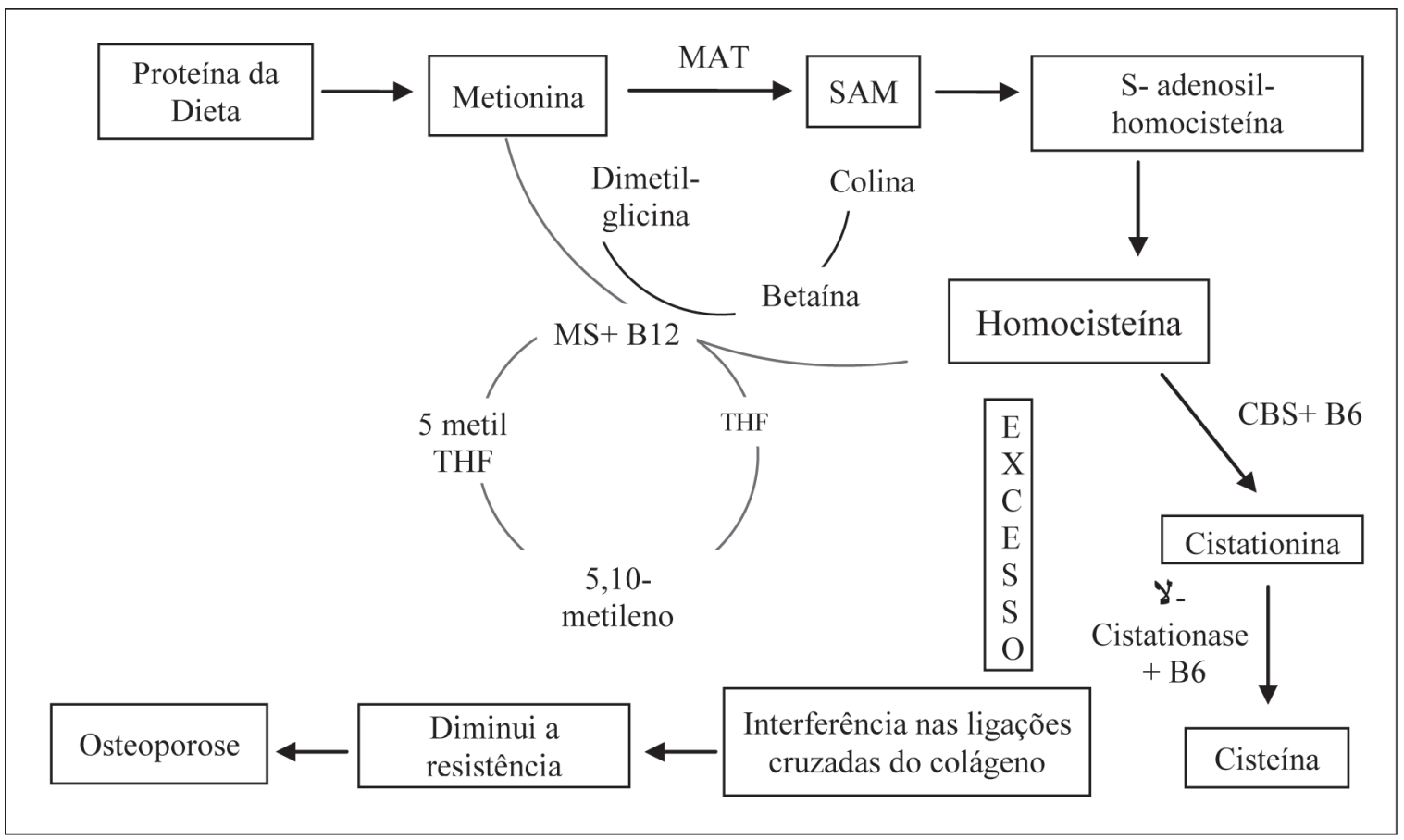

Figura 1 - Representação ilustrativa do metabolismo da homocisteína e o provável mecanismo de desenvolvimento da osteoporose.

MAT $=$ Metionina adenosil-transferase SAM $=$ S- adenosil-metionina $; \mathrm{CBS}=$ cistationa $\mathrm{B}$ sintetase; $\mathrm{TFH}=$ tetrahidrofolato; $\mathrm{MS}=$ metionina-sintetase.

As alterações ocorridas no metabolismo da homocisteína são de causa multifatorial, envolvendo fatores genéticos, fisiológicos, nutricionais e hormonais.

\section{Associação entre a deficiência de vitamina B12, folato, vitamina} B6 e da hiper-homocisteinemia com a diminuição da massa óssea

No Horland Homocysteine Study, que contou com a participação de 4.766 idosos, Gjesdal et al. ${ }^{11}$ observaram que mulheres com baixos níveis séricos de folato e elevadas concentrações de homocisteína tinham mais probabilidade de apresentar fratura de quadril. No mesmo estudo, a hiper-homocisteinemia foi considerada fator de risco para fraturas em ambos os sexos. Além dos achados citados, os autores verificaram que a presença do genótipo TT do gene MTHFR, em idosos com baixas concentrações de folato, aumentava em duas vezes as chances de fraturas, quando comparados com aqueles com genótipo $\mathrm{VV}$, que tinham elevados níveis de folato.
$\mathrm{Na}$ etapa seguinte do Horland Homocysteine Study, entre 1997 e 2000, Gjesdal et al. ${ }^{10}$ constataram que elevados níveis de homocisteína e baixos níveis de folato diminuem a DMO em mulheres, mas não em homens. Observaram também que a ingestão de cálcio e vitamina $\mathrm{D}$, embora tenha uma importante participação na formação e manutenção da matriz óssea, não modificava os índices de perda óssea, quando ocorria somente no período da menopausa. Esse estudo não encontrou associação entre o déficit de vitamina B12, polimorfismo no gene MTHFR e a perda de massa óssea.

Os achados de McLean et al., ${ }^{25}$ ao avaliarem 1.002 idosos participantes do Framingham Osteoporosis Study, mostraram que as baixas concentrações de vitamina B6 precipitavam as perdas ósseas e representavam fator de risco para fraturas de quadril. A carência de vitamina B12 associava-se às fraturas e a hiperhomocisteinemia aumentava em $69 \%$ as chances de fraturas de quadril. 
Baines et al., ${ }^{15}$ em estudo envolvendo 328 mulheres britânicas na pós-menopausa, observaram que os baixos níveis séricos de folato têm forte relação com a osteoporose. Já o déficit de vitamina B12 e de vitamina B6 tem uma relação mais estreita com os níveis de homocisteína, podendo exercer efeito deletério sobre o osso, embora menos pronunciado que o folato. Os autores concluíram também que o tabagismo estava associado com uma diminuição na $\mathrm{DMO}$ e diminuição do peso, além de reduzir os níveis de vitamina B6 e vitamina B12.

Tucker et al. ${ }^{26}$ examinaram a relação entre vitamina B12 e DMO nos participantes do Framingham Offspring Osteoporosis Study e concluíram que as concentrações séricas de vitamina B12 inferiores a 148 pM tinham relação com maior perda de massa óssea. Os autores também observaram que, nas mulheres, essas perdas se mostraram mais acentuadas na coluna vertebral, enquanto que nos homens se concentravam mais no quadril.

Bozkurt et al. ${ }^{27}$ avaliaram 178 mulheres turcas na pós-menopausa e concluíram que pacientes com diagnóstico de osteoporose na coluna lombar e no fêmur proximal ao DXA (dual energy X-ray absiorptiometry) apresentavam elevados níveis de homocisteína e baixos níveis de vitamina B12, representando, deste modo, fator de risco para fraturas nessa população. Uma pesquisa envolvendo 161 mulheres italianas na pósmenopausa demonstrou que o folato se relaciona positivamente com a mineralização óssea, ao contrário da vitamina B12 e da homocisteína. ${ }^{28}$

Tabela 1 - Principais estudos sobre vitamina $B_{12}$, folato e vitamina $B_{6}$ e sua associação com a massa óssea. Porto Alegre, RS, 2010.

\begin{tabular}{|c|c|c|}
\hline Autor & População & Resultados \\
\hline Baines et al. ${ }^{15}$ & 328 mulheres britânicas & $\begin{array}{l}\text { - Baixos níveis séricos de folato } \\
\text { relacionaram-se com a osteoporose. } \\
\text { - Baixos níveis séricos de vitamina } \mathrm{B}_{12} \text { e } \\
\text { vitamina } \mathrm{B}_{6} \text { relacionam-se com alterações } \\
\text { nos níveis séricos de homocisteína. }\end{array}$ \\
\hline McLean at al. ${ }^{25}$ & $\begin{array}{l}\text { - Framingham Osteoporosis Study } \\
\text { - } 1.002 \text { idosos }\end{array}$ & $\begin{array}{l}\text { - Deficiência de vitamina } \mathrm{B}_{6} \text { precipitava as } \\
\text { perdas ósseas. } \\
\text { - Deficiência de vitamina } \mathrm{B}_{12} \text {, aumentou o } \\
\text { rico de fratura de quadril. }\end{array}$ \\
\hline Gjesdal et al. ${ }^{11}$ & $\begin{array}{l}\text { - Horland Homocysteine Study } \\
\text { - } 4.766 \text { idosos }\end{array}$ & $\begin{array}{l}\text { - Mulheres com baixos níveis de folato e } \\
\text { elevadas concentrações de homocisteína } \\
\text { apresentaram uma maior probabilidade de } \\
\text { fratura de quadril. } \\
\text { - A hiper-homocisteína representou um } \\
\text { fator de risco para fratura de quadril em } \\
\text { ambos os sexos. }\end{array}$ \\
\hline $\begin{array}{l}\text { Dhonukshe- } \\
\text { Rutten et al. } .^{30}\end{array}$ & - 194 idosos & $\begin{array}{l}\text { - Mulheres com osteoporose tinham baixos } \\
\text { níveis de vitamina B12 e elevados níveis de } \\
\text { ácido metilmalônico. } \\
\text { - Nos homens essa associação não se } \\
\text { mostrou significante. }\end{array}$ \\
\hline
\end{tabular}


Em outro estudo envolvendo 194 idosos, Dhonukshe-Rutten et al. ${ }^{29}$ verificaram que mulheres com osteoporose tinham baixos níveis de vitamina B12 e elevados níveis de ácido metilmalônico, importante marcador da deficiência de vitamina B12. No entanto, essa associação não se mostrou significativa para homens. Morris et al. ${ }^{30}$ investigaram mulheres americanas participantes do NHANES III e também constataram que as baixas concentrações de vitamina B12, juntamente com elevados níveis de ácido metilmalônico, precipitavam as perdas ósseas.

No Longitudinal Aging Study Amsterdam (LASA), os resultados encontrados sugerem que as concentrações séricas de vitamina B12 abaixo de $200 \mathrm{Mp}$ eram fator de risco para as fraturas, mais em mulheres em relação aos homens. Também foi demonstrado que a homocisteína, em níveis séricos acima de $15 \mu \mathrm{M}$, estava associada mais a fraturas em homens do que em mulheres. Já as baixas concentrações de vitamina B12 e o aumento da homocisteína estavam relacionados a valores diminuídos de massa óssea à ultrassonometria de calcâneo e aumentados nos marcadores do turnover ósseo, deoxipirinolina/ creatinina (DPD/Cr) e osteocalcina, indicando, desta forma, que a formação óssea pode ser afetada pelas baixas concentrações de vitamina B12 e altas de homocisteína. ${ }^{16}$

No Osteoporosis Prospective Risk. Assessment (OPRA), que contou com a participação de 996 mulheres com idade acima de 75 anos, verificouse que altos níveis de homocisteína aumentavam as concentrações de telopeptídeos do colágeno tipo I (S-CTX), deoxipirinolinas urinárias (U-DPD) e osteocalcina. Além desses achados, observou-se que com a presença de hiperhomocisteinemia houve redução na densidade mineral óssea do fêmur ao DXA e diminuição em $2 \%$ na ultrassonometria de calcâneo. Dessa forma, constatou-se que a hiper-homocisteína está associada com o aumento do turnover ósseo e, consequentemente, com diminuição da DMO. ${ }^{31}$

$\mathrm{Na}$ Alemanha, em estudo envolvendo 94 indivíduos de ambos os sexos, submetidos à artroplastia de quadril por osteoartrite, recrutados pelo Orthopedic Department of the Knappschaftskrankenhaus Puttlingen, os autores observaram que indivíduos com elevadas concentrações séricas de vitaminas do complexo B apresentavam elevadas concentrações de osteocalcina, que é um importante marcador de formação óssea. Os achados desse estudo também indicaram que as baixas concentrações séricas de folato e vitamina B6 alteravam a estrutura do osso esponjoso, mas tal associação não se mostrou significativa quando relacionada com a vitamina B12. Já os resultados da análise histomorfométrica indicaram que indivíduos com baixas concentrações séricas de folato apresentaram tanto uma densidade quanto uma área trabecular diminuída. Dessa forma, os autores concluíram que as alterações na estrutura do osso esponjoso se explicariam pela diminuição da atividade osteoblástica, por meio das baixas concentrações de osteocalcina, em indivíduos com baixos níveis séricos de folato e vitamina B6. ${ }^{32}$

Dentro do processo metabólico ósseo, os osteoclastos, que são o grupo celular responsável pela reabsorção óssea, excretam TRACP5b, que é um importante marcador da reabsorção óssea. Assim, com a presença de altos níveis de homocisteína, eleva-se a quantidade de TRACP5b, indicando que a atividade osteoclástica aumenta com as altas concentrações de homocisteína. ${ }^{33-35}$ A hiper-homocisteinemia leva a um desequilíbrio entre a atividade dos osteoblastos e osteoclastos, com maior intensidade nestes últimos. ${ }^{6} \mathrm{~A}$ deficiência de vitamina B12 diminui a massa óssea, pois eleva os níveis de homocisteína e o ácido metilmalônico, aumentando a formação de osteoclastos. ${ }^{36}$

Os níveis de homocisteína podem ser alterados por uma mutação no gene C677T da MTHFR, que induz a substituição de uma valina por alanina. Essa condição reduz a atividade enzimática da MTHFR, aumentando os níveis de homocisteína. ${ }^{37}$ Estudo realizado com 307 mulheres japonesas verificou associação entre a diminuição da DMO na presença do genótipo 
$V V$ da MTHFR. ${ }^{38}$ Por outro lado, estudo envolvendo 1.748 mulheres participantes do Danish Osteoporosis Prevention Study constatou que mulheres portadoras do genótipo TT tinham diminuição da DMO de fêmur, quadril e coluna vertebral, além de apresentarem duas vezes mais risco de fraturas em relação àquelas com genótipos CC ou CT. ${ }^{39}$

Já nos achados de Hong et al., ${ }^{37}$ não houve associação entre o genótipo C677T da MTHFR e a baixa DMO em mulheres chinesas; entretanto, os portadores do alelo $\mathrm{T}$ apresentaram maior risco de fraturas, independentemente de estarem ou não na menopausa. Recentemente, verificouse que o folato é um dos principais fatores que poderiam explicar a variação na densidade mineral óssea da coluna lombar e fêmur proximal em mulheres iranianas, sugerindo, desta forma, que a hiper-homocisteinemia pode estar relacionada de forma mais estreita com a redução do folato, mais do que pela presença do polimorfismo do gene MTHFR. ${ }^{40}$

$\mathrm{Na}$ Eslováquia, pesquisa envolvendo 140 mulheres com dieta ovo-lacto-vegetariana e 131 mulheres com dieta ocidental padrão verificou que mulheres usuárias de dieta vegetariana apresentavam níveis aumentados de homocisteína e paratormônio (PTH), além de baixa $\mathrm{DMO}$ nas regiões anatômicas do trocânter e fêmur total. Esses dados demonstram que a hiperhomocisteinemia poderia ser importante fator de risco para a perda de massa óssea, sobretudo em mulheres com dieta vegetariana. ${ }^{41}$

Sato et al. ${ }^{17}$ analisaram 629 pacientes com hemiplegia pós-acidente vascular cerebral (AVC), usuários de um serviço hospitalar japonês, no período de 2000 a 2001, sendo submetidos ao uso de $1.500 \mu \mathrm{g}$ de vitamina B12 e $5 \mathrm{mg}$ de folato diariamente. Os resultados indicaram que a suplementação com vitaminas $\mathrm{B} 12$ e folato reduziu o risco de fraturas em pacientes pós-AVC. Green et al. ${ }^{42}$ suplementaram $100 \mu \mathrm{g}$ de folato, $500 \mu \mathrm{g}$ de vitamina B12 e $10 \mathrm{mg}$ de vitamina $\mathrm{B} 6 \mathrm{em}$ idosos e concluíram que a suplementação destes micronutrientes reduzia os níveis de homocisteína, mas não alterava os marcadores de formação e reabsorção óssea.

Diferentes hipóteses tentam explicar a relação entre homocisteína e fraturas ósseas, no entanto, a mais citada é a proposta por McKusick, ${ }^{43}$ que sugere que a homocisteína interfere nas ligações cruzadas do colágeno, influenciando na formação óssea. Em estudo realizado com animais que receberam dieta rica em metionina e homocisteína, evidenciou-se que os elevados níveis de homocisteína dificultavam a capacidade de metilação e que $65 \%$ do excesso de homocisteína se encontravam no colágeno extracelular, sendo que essas elevadas concentrações caracterizaram uma redução do osso esponjoso com diminuição da força óssea ${ }^{44}$

\section{CONCLUSÃO}

O processo de envelhecimento torna o idoso mais suscetível a carências nutricionais, inclusive vitamínicas. A deficiência de vitamina B12, vitamina B6 e folato acomete frequentemente a população idosa, sendo que esses déficits têm uma repercussão em parâmetros hematológicos, neurológicos, cardiovasculares e também no tecido ósseo, tornando o idoso mais propenso a osteoporose e fraturas ósseas por fragilidade.

De acordo com a presente revisão, podese verificar que os elevados níveis séricos de homocisteína representam fator de risco para a integridade óssea, seja por deficiência de vitamina B12, vitamina B6 e folato, ou mutações genéticas. Esse aminoácido sulfurado, quando em concentrações elevadas, promove interferências nas ligações cruzadas do colágeno, ocasionando alterações na estrutura óssea.

Neste contexto, embora diversos estudos evidenciem a importância dessas vitaminas para o metabolismo ósseo, estudos em diferentes grupos populacionais, inclusive brasileiros, se fazem necessários para a melhor compreensão dessa relação. 


\section{REFERÊNCIAS}

1. Lourenço RA, Martins CFM, Sanchez MAS, Veras PP. Assistência ambulatorial geriátrica: hierarquização da demanda. Rev .Saude Publ 2005;39(2):311-8.

2. Lloyd-Sherlock P. Population ageing in developed and developing regions: implications for health policy. Soc Sci Med 2000; 51:887-95.

3. Parahyba MI, Wallace A. Síntese de indicadores sociais - 2002. In: Fundação IBGE. Departamento de População e Indicadores Sociais. Idosos. Rio de Janeiro; 2003.

4. Carvalho CMRG, Fonseca CGC, Pedrosa JI. Educação para a saúde em osteoporose com idosos de um programa universitário: repercussões. Cad Saúde Pública 2004 Mai./Jun; 20(3): 719-26.

5. Lippuner K, Golder M, Greiner R. Epidemiology and direct medical costs of osteoporotic fractures in men and women in Switzerland. Osteoporos Int 2000 Mar; 16 suppl 2: S8-S17.

6. Herrmann M, Umanskaya N, Wildemann B, Colaianni G, Widmann T, Zallone A, et al. Stimulation of osteoblast activity by homocysteine. J Cell Mol Med 2008 Aug; 12(4): 1205-10.

7. Kenny AM, Prestwood KM. Osteoporosis. Pathogenesis, diagnosis and treatment in older adults. Rheum Dis Clin North Am 2000 Aug; 26(3): 569-91.

8. Yazbek MA, Neto JFM. Osteoporose e outras doenças osteometabólicas no idoso. Einstein 2008; 6 suppl 1: S74-S78.

9. Wilkins $\mathrm{CH}$, Birge, SJ. Prevention of osteoporotic fractures in the elderly. Am J Med 2005 Nov; 118(11): 1190-5.

10. Gjesdal CG, Vollset SE, Ueland PM, Refsum H, Drevon CA, Gjessing HK, et al . Plasma total homocysteine level and bone mineral density: the Hordaland Homocysteine Study. Arch Intern Med 2006 Jan; 166(1): 88-94.

11. Gjesdal CG, Vollset SE, Ueland PM, Refsum H, Drevon CA, Meyer HE, Tell GS. Plasma homocysteine, folate, and vitamin B12 and the risk of hip fracture: the Hordaland Homocysteine Study. J Bone Miner Res 2007 May; 22(5): 747-56.

12. McLean RR, et al. Homocysteine as a predictive factor for hip fracture in older persons. N Engl J Med 2004 May; 350(20): 2042-9.

13. Walker-Bone K, Walter G, Cooper C. Recent developments in the epidemiology of osteoporosis. Curr Opin Rheumatol 2002 Jul; 14(4): 411-5.
14. Froes NDTC, Pereira ES, Negrelli WF. Fatores de risco da osteoporose: prevenção e detecção através do monitoramento clínico e genético. Acta Ortop Bras 2002 Jan./Mar; 10(1):52-7.

15. Baines $\mathrm{M}$, et al. The association of homocysteine and its determinants MTHFR genotype, folate, vitamin $\mathrm{B} 12$ and $\mathrm{B} 6$ with bone mineral density in postmenopausal British women. Bone 2007 Mar; 40(3): 730-6.

16. Dhonuskshe- Rutten RA, Pluijm SM, de Groot LC, Lips P, Smit JH, van Staveren WA. et al. Homocysteine and vitamin B12 status relate to bone turnover markers, broadband ultrasound attenuation, and fractures in healthy elderly people. J Bone Miner Res 2005 Jun; 20(6): 921-9.

17. Sato Y, Honda Y, Iwamoto J, Kanoko T, Satoh, K, et al. Effect of folate and mecobalamin on hip fractures in patients with stronke: A randomized controlled trial. JAMA 2005 mar; 293(9): 1082-8.

18. McLean RR, Hannan MT. B vitamins, homocysteine, and bone disease: epidemiology and pathophysiology. Curr Osteoporos Rep 2007Sep; 5(3): 112-9.

19. Bydlowski SP, Magnanelli AC, Chamone DAF. Hiper-homocisteinemia e doenças vaso-oclusivas. Arq. Bras. Cardiol 1998 July; 71(1): 69-76.

20. Deminice R, Vilhena R, Portari GV, Jordão AA. Suplementação de creatina, homocisteína e estresse oxidativo.Medicina (Ribeirão Preto) 2007;40 (3): 368 77 , jul./set.

21. Neves LB, Macedo DM, Lopes AC. Homocisteína. J Bras Patol Med Lab 2004 Out; 40(5): 311-20.

22. Baines M, Kredan MB, Davison A, Higgins G, West C, Fraser WD, et al. The association between cysteine, bone turnover, and low bone mass. Calcif Tissue Int 2007 Dec; 81(6): 450-4.

23. Cashman KD. Homocysteine and osteoporotic fracture risk: a potential role for B vitamins. Nutr Rev 2005 jan; 63(1): 29-36.

24. Liu G, Nellaiappan K, Kagan HM. Irreversible inhibition of lysyl oxidase by homocysteine thiolactone and its selenium and oxygen analogues: implications for homocystinuria. J Biol Chem 1997 Dec; 272(51): 32370-7.

25. McLean RR, Jacques PF, Selhub J, Fredman L, Tucker KL, Samelson EJ,et al. Plasma B vitamins, homocysteine, and their relation with bone loss and hip fracture in elderly men and women. Clin Endocrinol Metab 2008 Jun; 93(6): 2206-12. 
26. Tucker KL, Jugdaohsingh R, Powell JJ, Quiao N, Hannan MT, Sripanyakorn S, et al. Low plasma vitamin B12 is associated with lower BMD: The Framingham Osteoporosis Study. J Bone Miner Res 2005 Jan; 20(1): 152-8.

27. Bozkurt N, Erdem M, Yilmaz E, Erdem A, Biri A, Kubatova A,et al. The relationship of homocyteine, B12 and folic acid with the bone mineral density of the femur and lumbar spine in Turkish postmenopausal women. Arch Gynecol Obstet 2009 Sep; 280(3): 381-7.

28. Cagnacci A, Baldassari F, Rivolta G, Arangino S, Volpe A. Relation of homocysteine, folate, and vitamin B12 to bone mineral density of postmenopausal women. Bone 2003 Dec; 33(6): 956-959.

29. Dhonukshe-Rutten RA, Lips M, Jong N, Chin A Paw MJM, Hiddink GJ, van Dusseldorp M, et al. Vitamin B-12 status is associated with bone mineral content and bone mineral density in frail elderly women but not in men. J. Nutr 2003 Mar; 133(3): 801-7.

30. Morris MS, Jacques PF, Selhub J. Relation between homocysteine and B-vitamin status indicators and bone mineral density in older Americans. Bone 2005 Aug; 37(2): 234-42.

31. Gerdhem P, Ivaska KK, Isaksson A, Pettersson $\mathrm{K}$, Vaananen Hk, Obrant HJ, et al. Associations between homocysteine, bone turnover, BMD, mortality, and fracture risk in elderly women. J Bone Miner Res 2007 Jan; 22(1): 127-34.

32. Holstein JH, Herrmann M, Splett C, Hermann W, Garcia P, Histing T,et al. Low serum folate and vitamin B-6 are associated with an altered cancellous bone structure in humans. Am J Clin Nutr 2009 Nov; 90:(5) 1440-5.

33. Herrmann M, Widmann T, Colaianni G, Colucci S, Zallone A, Herrmann W. Increased osteoclast activity in the presence of increased homocysteine concentrations. Clin Chem 2005 Dec; 5(12): 2348-53.

34. Nenonen A, et al. Serum TRACP 5b is a useful marker for monitoring alendronate treatment: comparison with other markers of bone turnover. J Bone Miner Res 2005 Oct; 20(10): 1804-12.

35. Janckila AJ, Takahashi K, Susan Z, Yam SLT. Tartrate-resistant acid phosphatase isoform $5 \mathrm{~b}$ as serum marker for osteoclastic activity. Clin Chem 2001Jan; 47(1): 74-80.
36. Vaes BL, Lute C, Blom HJ, Bravemboer N, de Vries TJ, Everts V, et al. Vitamin B12 deficiency stimulates osteoclastogenesis via increased homocysteine and methylmalonic acid. Calcif Tissue Int 2009 May; 84(5):413-22.

37. Hong X, Hsu YH, Terwedow H, Tang G, Liu X, Jiang $\mathrm{S}$, et al. Association of the Methylenetetrahydrofolate reductase $\mathrm{C} 677 \mathrm{~T}$ polymorphism and fracture risk in Chinese postmenopausal Women. Bone 2007 Mar; 40(3): 737-42.

38. Miyao M, Morita H, Hosoi T, Kurihara H, Inoue S, Hoshino S,et al. Association of methylenetetrahydrofolate reductase (MTHFR) polymorphism with bone mineral density in postmenopausal Japanese women. Calcif Tissue Int 2000 Mar; 66(3): 190-4.

39. Abrahamsen B, Madsen JS, Tofteng CL, Stilgren L, Bladbjerg EM, Kristensen SR. A common methylenetetrahydrofolate reductase (C677T) polymorphism is associated with low bone mineral density and increased fracture incidence after menopause: longitudinal data from the Danish osteoporosis prevention study. J Bone Miner Res 2003 Apr; 18(4): 723-9.

40. Golbahar J, Hamidi A, Aminzadeh MA, Omrani GR. Association of plasma folate, plasma total homocysteine, but not methylenetetrahydrofolate reductase C667T polymorphism, with bone mineral density in postmenopausal Iranian women: a crosssectional study. Bone 2004 Sep; 35(3): 760-5.

41. Krivosíková Z, Krajcovicová-Kudlácková M, Spustová V, et al. The association between high plasma homocysteine levels and lower bone mineral density in Slovak women: the impact of vegetarian diet. Eur J Nutr 2010 Apr; 49(3): 147-53.

42. Green TJ, McMahon JA, Skeaff CM, Williams SM, Whiting SJ. Lowering homocysteine with B vitamins has no effect on biomarkers of bone turnover in older persons: a 2-y randomized controlled trial. Am J Clin Nutr 2007Febr; 85(2): 460-4.

43. McKusick VA: Heritable disorders of connective tissue. St Louis: C.V. Mosby; 1966.

44. Herrmann M, Tami A, Wildemann B, Wolny M, Wagner A, Schorr H,. Hyperhomocysteinemia induces a tissue specific accumulation of homocysteine in bone by collagen binding and adversely affects bone. Bone 2009 Mar; 44(3): 467-75. 